\title{
BCR-JAK2 Fusion Protein Expression
}

National Cancer Institute

\section{Source}

National Cancer Institute. BCR-JAK2 Fusion Protein Expression. NCI Thesaurus. Code C37888.

Expression of a fusion protein involving the human genes BCR and JAK2. It results from a translocation and is associated with atypical chronic myeloid leukemia, myleoproliferative neoplasms, acute myeloid leukemia, B lymphoblastic leukemia/lymphoma and Burkitt lymphoma. 